\title{
Politique de la drogue: oui à une solution qui a fait ses preuves, pas de consensus sur la solution nouvelle
}

Les images du «Platzspitz» à Zurich ont fait la une de journaux et le tour du monde: des toxicomanes hagards titubaient au petit matin devant des citadins en promenade avec leur chien; on y trouvait des seringues utilisées on parlait déjà du sida - près des places de jeux pour les enfants; on y croisait des «junkies» en train de mendier, clairement agressifs lorsqu'ils étaient en manque. Le tout accompagné d'un débat idéologique ne menant à rien. Jusqu'au jour où, sous la pression de cette misère omniprésente, l'idée s'est imposée progressivement que toute personne a droit à un soutien médical et social, même lorsqu'elle n'est pas en mesure de renoncer à consommer des drogues illégales.

A la fin des années 80 s'est amorcée une évolution dont le scrutin du 30 novembre 2008 devrait permettre d'ancrer les fruits dans la loi: la politique dite des quatre piliers, qui combine prévention, thérapie, réduction des risques et répression.

Cette politique comprend un large éventail de mesures et d'offres. Ainsi, la remise de seringues à usage unique, de même que les locaux d'injection permettant une consommation «sécurisée», qui firent l'objet de débats houleux à l'époque, ont permis d'influencer positivement le taux de nouvelles infections par VIH. Grâce à des offres médicales à bas seuil, l'état de santé des toxicomanes s'est amélioré. Des projets d'habitat et de travail avec encadrement ont rendu possible une meilleure intégration. Soins et accompagnement ont ouvert de nouvelles perspectives à des milliers d'êtres humains.

Les chiffres sont impressionnants: 1500 thérapies résidentielles, 17500 traitements de substitution à la méthadone, 1500 personnes gravement toxicodépendantes suivant un traitement avec prescription d'héroïne et environ 20000 autres bénéficiant d'un traitement ambulatoire: tous ces gens ne sont plus dans la rue mais ont la possibilité de se stabiliser socialement, d'assumer la responsabilité de leur existence et de réintégrer la société.

La mise en œuvre des quatre piliers - un véritable succès - a valu à la politique suisse en matière de drogue des échos très positifs dans les médias, notamment sur le plan international. Un non dans les urnes nous ramènerait à un débat politique sur la drogue marqué par les idéologies. Refuser la politique des quatre piliers, ce serait succomber à l'illusion d'une société sans drogues qu'on puisse imposer par la force si nécessaire. Or il n'y a nulle part au monde de signes indiquant que des solutions basées sur la seule répression puissent fonctionner.

Cette constatation vaut aussi pour le cannabis: la répression à elle seule n'a aucun effet! L'initiative pour le chanvre propose un chemin pour sortir d'une voie sans issue. L'existence de quelque 500000 consommateurs occasionnels ou réguliers de cannabis montre clairement que le problème ne peut être résolu par des mesures policières. Nous avons besoin de solutions nouvelles: il convient de protéger la jeunesse et de dépénaliser la consommation, sans pour autant minimiser les risques liés à la drogue.

Toutefois, l'initiative pour le chanvre, sur laquelle le peuple suisse se prononcera aussi le 30 novembre, n'est pas parvenue à convaincre les délégué-e-s de la FMH. Lors de l'Assemblée des délégués du 4 septembre 2008, ils ont maintenu leur décision de laisser la liberté de vote sur cet objet. En disant clairement oui à la loi sur les stupéfiants, la FMH dit oui à une solution qui a fait ses preuves. En revanche, la liberté de vote pour l'initiative sur le chanvre est à voir comme l'expression d'un malaise persistant. Personne ne conteste que la situation actuelle soit insatisfaisante, mais il n'y a pas encore de consensus sur la façon de sortir de l'impasse. La discussion sur le cannabis a encore un long chemin à parcourir, au sein de la société toute entière, pour se défaire des considérations idéologiques, des préjugés et des peurs, et devenir un débat objectif et fondé sur des faits qui permettra de développer des solutions pratiques et susceptibles de consensus.

Dr Christine Romann, membre du Comité central de la FMH, responsable du domaine Promotion de la santé et prévention 